\title{
Treatment of autoimmune hemolytic anemia: real world data from a reference center in Mexico
}

\author{
José Carlos Jaime-Pérez, Patrizia Aguilar-Calderón, Lorena Salazar-Cavazos, Andrés Gómez-De León, \\ David Gómez-Almaguer \\ Department of Hematology, Internal Medicine Division, Dr. José E. González University Hospital, School of Medicine of the \\ Universidad Autónoma de Nuevo León, Monterrey, Mexico
}

p-ISSN 2287-979X / e-ISSN 2288-0011 https://doi.org/10.5045/br.2019.54.2.131

Blood Res 2019;54:131-136.

Received on November 24, 2018

Revised on February 12, 2019

Accepted on March 23, 2019

\section{Correspondence to}

José Carlos Jaime-Pérez, M.D., Ph.D.

Hospital Universitario Dr. José E. González, Hematología, Edificio "Dr. Rodrigo

Barragán Villarreal" $2^{\circ}$ piso Ave. Maderoy Ave. Gonzalitos s/n, Colonia Mitras Centro, CP 64460 Monterrey, N.L. Mexico E-mail: carjaime@hotmail.com

(C) 2019 Korean Society of Hematology

\section{Background}

Warm autoimmune hemolytic anemia (w-AIHA) is an uncommon disease with heterogeneous response to treatment. Steroids are the standard treatment at diagnosis, whereas rituximab has recently been recommended as the second-line therapy of choice. Our main objective was to document the response to treatment in patients with newly diagnosed w-AIHA, including the effectiveness of low-dose rituximab as frontline treatment and for refractory disease.

\section{Methods}

Patients with w-AIHA from 2002 to 2017 were included. Relapse-free survival (RFS), probability of maintained response (MR), and time-to-response were analyzed using the Kaplan-Meier method. Response was classified as complete, partial, and no response.

\section{Results}

We included 64 adults with w-AIHA (39 women and 25 men). The median age was 37 $(16-77)$ years. Response rates to steroids alone were $76.7 \%$, rituximab plus steroids, $100 \%$; and cyclophosphamide, $80 \%$. RFS with steroids at 6, 36, and 72 months was $86.3 \%$, $65.1 \%$, and $59.7 \%$, respectively. Eighteen patients received rituximab at $100 \mathrm{mg} / \mathrm{wk}$ for 4 weeks plus high-dose dexamethasone as first-line therapy, with RFS at 6, 36, and 72 months of $92.3 \%, 58.7 \%$ and $44.1 \%$, respectively. Eight patients refractory to several lines of therapy were treated with low-dose rituximab, and all achieved a response (three complete response and five partial response) at a median 16 days (95\% confidence interval, 14.1-17.8), with a 75\% probability of MR at 103 months; the mean MR was $81.93 \pm 18$ months.

\section{Conclusion}

Outcomes of w-AlHA treatment were considerably heterogeneous. Low rituximab doses plus high dexamethasone doses were effective for refractory disease.

Key Words Autoimmune hemolytic anemia, Low-dose rituximab, Refractory autoimmune hemolytic anemia, Steroids, Splenectomy, Immune cytopenia

\section{INTRODUCTION}

Autoimmune hemolytic anemia (AIHA) is an infrequent disease characterized by the self-destruction of red blood cells mediated by autoantibodies (anti-RBC). World incidence of AIHA is about $1-3$ per 100,000 population. AIHA is classified as warm, cold, mixed, and drug-induced [1]. This study focused on the warm type, which represents $80 \%$ of all cases of AIHA [2]. The pathogenesis of this disease is still uncertain, involving a complex process intertwining autoantigens, the complement system, and abnormalities of $\mathrm{T}$ and $\mathrm{B}$ cells.

The diagnosis of w-AIHA is based on clinical characteristics and laboratory findings including anemia, hemolysis features such as elevated serum indirect bilirubin levels, low serum haptoglobin levels, increased lactate dehydrogenase (LDH) levels, elevated reticulocyte counts, and microscopic 
examination of a peripheral blood smear and positive direct antiglobulin test (DAT) using Coombs reagent. Hereditary hemolytic anemia and other diseases associated with autoimmune hemolysis should be ruled out [3].

Steroids remain the mainstay of therapy, being effective in $70-80 \%$ of patients [4-6]. Recently rituximab has emerged as an additional resource with fewer secondary effects than traditional immunosuppressive agents, which has led to this biological agent being formally recommended for second line therapy, displacing splenectomy. However, there is no evidence-based therapeutic regimen for this heterogeneous disease; thus, its management depends on individual patient characteristics, its etiology, laboratory results, and the experience of the physician [7]. We documented the clinical and laboratory features of patients with w-AIHA attending an academic hematology reference center over a period of 15 years, to describe the clinical pathways and assess outcomes using the different available therapeutic modalities, including rituximab at $100 \mathrm{mg} / \mathrm{wk}$ for 4 weeks, as we recently reported in immune thrombocytopenia (ITP) [8, 9].

\section{MATERIALS AND METHODS}

This retrospective analysis included 64 consecutive unselected patients with newly diagnosed w-AIHA from 2002 to 2017 who had complete information in clinical files and electronic databases and were treated at the Department of Hematology of Dr. Jose Eleuterio González University Hospital, School of Medicine of the Autonomous University of Nuevo León in Monterrey, Mexico.

Patients were adults $\geq 16$ years of age. The diagnosis of w-AIHA was established based upon classic clinical signs of hemolysis including jaundice, asthenia, adynamia, fatigue, hepatosplenomegaly, and anemia with hemoglobin $(\mathrm{Hb}) \leq$ $11 \mathrm{mg} / \mathrm{dL}$; a positive DAT; and increased levels of indirect bilirubin, $\mathrm{LDH}$, and reticulocytes. In all patients, hereditary hemolytic disease was ruled out [10]. Data on RBC-bound immunoprotein, IgG and or complement, and antibody specificity were unavailable as only polyspecific anti-human globulin (Coombs) reagent is used in our public hospital blood bank. The protocol of this study was approved by the Institutional Review Board and Ethics Committee, with approval number HE17-00009.

\section{Definitions of response}

A complete response $(\mathrm{CR})$ was considered with $\mathrm{Hb} \geq 12.0$ $\mathrm{g} / \mathrm{dL}$ in women and $\geq 13.0 \mathrm{~g} / \mathrm{dL}$ in men [11], partial response (PR) with $\mathrm{Hb} \geq 10.0 \mathrm{~g} / \mathrm{dL}$ with persistent hemolysis or with a $\geq 2.0 \mathrm{~g} / \mathrm{dL}$ increment in basal $\mathrm{Hb}$ at diagnosis without reaching normal values; no response was assumed with an $\mathrm{Hb}$ increase $<2.0 \mathrm{~g} / \mathrm{dL}[6]$; The overall response rate (ORR) comprised the sum of the CR and PR. Relapse was defined as the reemergence of disease. Duration of response was defined as the initial response until relapse or death. Patients with refractory $\mathrm{w}$-AIHA were those who received at least two therapeutic lines with $\mathrm{Hb}<11 \mathrm{~g} / \mathrm{dL}$ prior to rituximab
[12]. Maintained response (MR) was the maintenance of response more than 6 months after treatment administration [12].

\section{Treatment}

All patients were treated with steroids at diagnosis of w-AIHA, prednisone at $1-2 \mathrm{mg} / \mathrm{kg} /$ day for 28 days or dexamethasone at $40 \mathrm{mg} /$ day for 4 days [13]. Eighteen patients additionally received rituximab at $100 \mathrm{mg} / \mathrm{wk}$ for 4 weeks, on days 1, 7, 14, and 21 as frontline therapy. If no response was achieved or relapse developed, second-line options included rituximab at $100 \mathrm{mg} / \mathrm{wk}$ for 4 weeks plus high-dose dexamethasone (HDD) $[14,15]$ and splenectomy.

Cyclophosphamide at $300 \mathrm{mg} / \mathrm{m}^{2} / \mathrm{wk}$ and azathioprine at $150 \mathrm{mg} /$ day were third-line treatments; a portion of patients received a combination of these drugs. The ORR was analyzed for each type of treatment.

Low-dose rituximab was administered owing to financial restrictions in our low-income, uninsured patient population, who pay for their treatment out of pocket.

\section{Statistical analysis}

Statistical analysis was carried out using IBM SPSS v. 22 (IBM Corp., Armonk, NY, USA). Categorical variables are displayed as absolute numbers and percentages, and comparisons were made with the Pearson chi-square test. Quantitative variables were analyzed using descriptive statistics including median and range, and the Mann-Whitney $\mathrm{U}$ test was used to evaluate comparisons between quantitative variables. RFS, MR, and time to response were evaluated using Kaplan-Meier analysis. RFS in months was defined as the date of onset until relapse. Duration of MR in months was defined as the maintenance of response for more than 6 months after starting treatment. Duration of response in days was calculated from the time of PR or CR until relapse or death. A $P$-value $<0.05$ was considered significant.

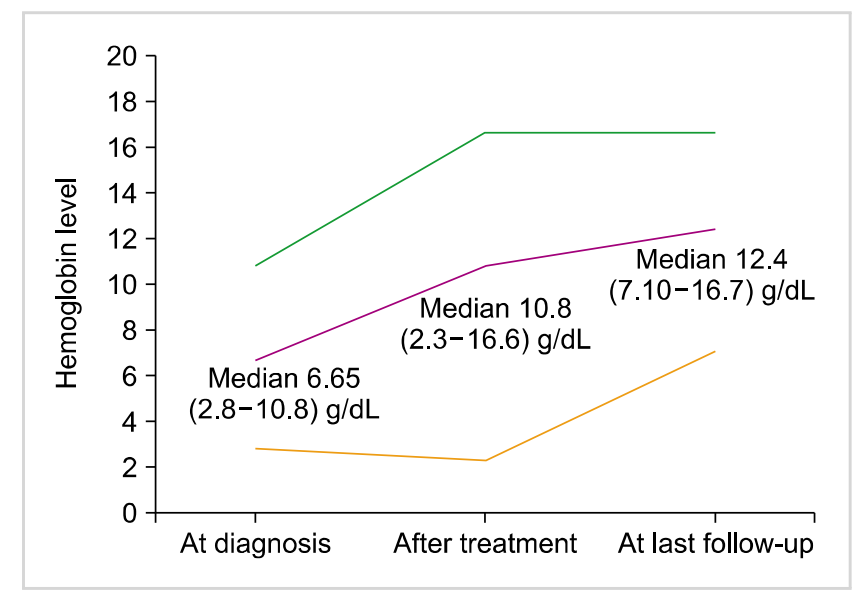

Fig. 1. Comparison between hemoglobin levels at diagnosis, after treatment and at last follow-up in 64 patients with w-AlHA, including median and ranges. 


\section{RESULTS}

\section{Patient characteristics and clinical features}

A total of 64 patients were included in this study over a period of 15 years. Thirty-nine $(60.9 \%)$ participants were women and 25 (39.1\%) were men; the median age at diagnosis was 37 (16-77) years. Clinical features were not associated with sex or etiology of w-AIHA; all patients had anemic syndrome.

An underlying disease was identified in 33 patients, including systemic lupus erythematosus (14 patients, 21.9\%); Evans syndrome (12, 18.8\%); antiphospholipid syndrome (2, 3.1\%); hyperthyroidism (2, 3.1\%); and chronic lymphocytic leukemia, Hodgkin lymphoma, and rheumatoid arthritis in 1 patient $(1.6 \%)$ each.

Median hemoglobin level at diagnosis was 6.65 (2.8-10.8) $\mathrm{g} / \mathrm{dL}$. Sequential median hemoglobin levels before and after treatment and at last follow-up are shown in Fig. 1. The remaining important laboratory characteristics are shown in Table 1.

Fifteen patients required transfusion owing to severity of anemic syndrome, with median $\mathrm{Hb} 5.6$ (2.8-9.3) g/dL. Two patients (3.12\%) died; one death was secondary to Kaposi sarcoma in an HIV-positive individual and another patient died after suffering an ischemic stroke associated with a hemolytic episode.

\section{First-line treatment}

In this cohort, 46 patients received steroids and 18 received low-dose rituximab plus HDD as first-line treatment. Of the group of patients who received steroids, an initial response was achieved in 36/46 (78\%) with a median time of 11.5 (1-30) days. There were 16 (35\%) patients with CR and $20(43 \%)$ with PR, with a median duration of response 22 (11-31) months. At last follow-up, 70\% of patients were in CR and $30 \%$ in PR. No difference according to type of steroid was observed $(P=0.679)$. Median RFS was 81.7 months [95\% confidence interval (CI) 71.41-88.57]; RFS at 6, 36, and 72 months was $86.3 \%, 65.1 \%$, and $59.7 \%(P=0.697)$ (Fig. 2).

All 18 patients (25\%) who received low-dose rituximab plus HDD as first-line therapy achieved an initial response with a median time of 14 (3-30) days, including 16 patients with PR and 2 with CR; the median duration of response was 16.5 (1-39) months. At last follow-up, 15 (83\%) patients were in CR and $3(17 \%)$ patients had PR. Median RFS was 33.1 months (95\% CI, 28.15-37.91); RFS at 6, 36, and 72 months was $92.3 \%, 58.7 \%$, and $44.1 \%(P=0.697)$ (Fig. 2). No adverse effects were documented in this group of patients.

\section{Rituximab in refractory w-AlHA}

Ten patients $(15.6 \%)$ in our cohort had refractory disease with a median 2.5 (2-4) lines of treatment including steroids

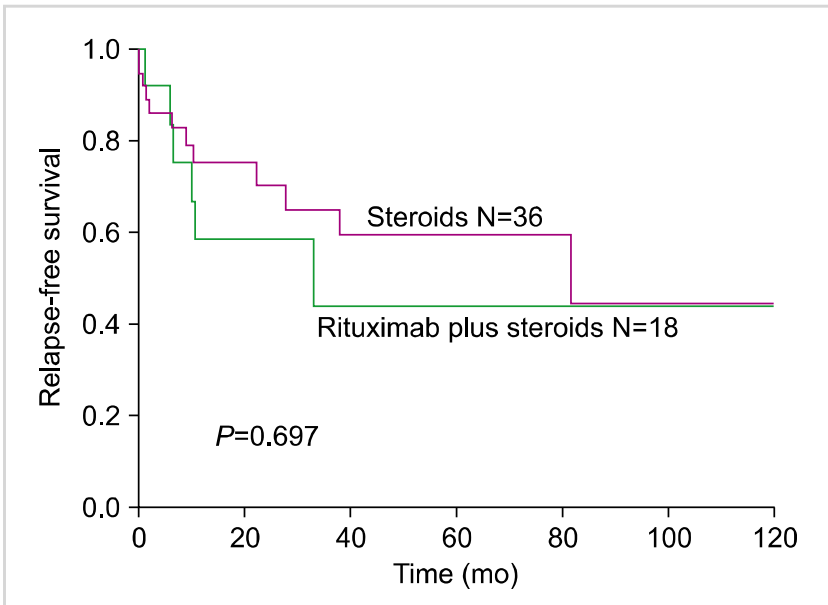

Fig. 2. Relapse-free survival in 54 patients who achieve a response treated with steroids only or rituximab at $100 \mathrm{mg} / \mathrm{wk} / 4$ weeks plus high doses of dexamethasone.

\begin{tabular}{|c|c|c|c|c|}
\hline Variable & & Steroids only, $N=46$ & $\begin{array}{l}\text { Low-dose rituximab } \\
\quad+\mathrm{HDD}, \mathrm{N}=18\end{array}$ & $P$ \\
\hline \multicolumn{5}{|l|}{ Sex } \\
\hline Female & $39(60.9 \%)$ & $28(60.9 \%)$ & $11(61.1 \%)$ & 0.460 \\
\hline Male & $25(39.1 \%)$ & $18(39.1 \%)$ & $7(38.9 \%)$ & 0.460 \\
\hline Age $(y r)$, median & $37(16-77)$ & $36.5(16-77)$ & $41.5(18-77)$ & 0.469 \\
\hline \multicolumn{5}{|l|}{ Laboratory features } \\
\hline Hemoglobin, g/dL & $6.65(1-10.8)$ & $6.48(2.8-10.8)$ & $5.5(1-10.2)$ & 0.547 \\
\hline Reticulocytes, \% & $8.17(0.03-65)$ & $6.4(0.03-65)$ & $17.3(1-54)$ & 0.445 \\
\hline $\mathrm{LDH}, \mathrm{IU} / \mathrm{L}$ & 434 (4.1-2938) & 439 (254-1630) & 340.5 (4.1-2938) & 0.417 \\
\hline Total bilirubin, mg/dL & $2.38(0.31-10.2)$ & $2.68(0.31-10.2)$ & $1.28\left(0.5^{-3.2)}\right.$ & 0.419 \\
\hline Indirect bilirubin, mg/dL & $1.74(0.4-9.8)$ & $2(0.4-9.8)$ & $1.2(0.59-2.8)$ & 0.460 \\
\hline Transfusion, RBCs & $15(23.4 \%)$ & $14(30.4 \%)$ & $1(5.5 \%)$ & 0.061 \\
\hline
\end{tabular}

Values presented in the table are $\mathrm{N}(\%)$ or value (range).

Abbreviations: HDD, high doses of dexamethasone; LDH, lactic dehydrogenase; RBCs, red blood cells. 

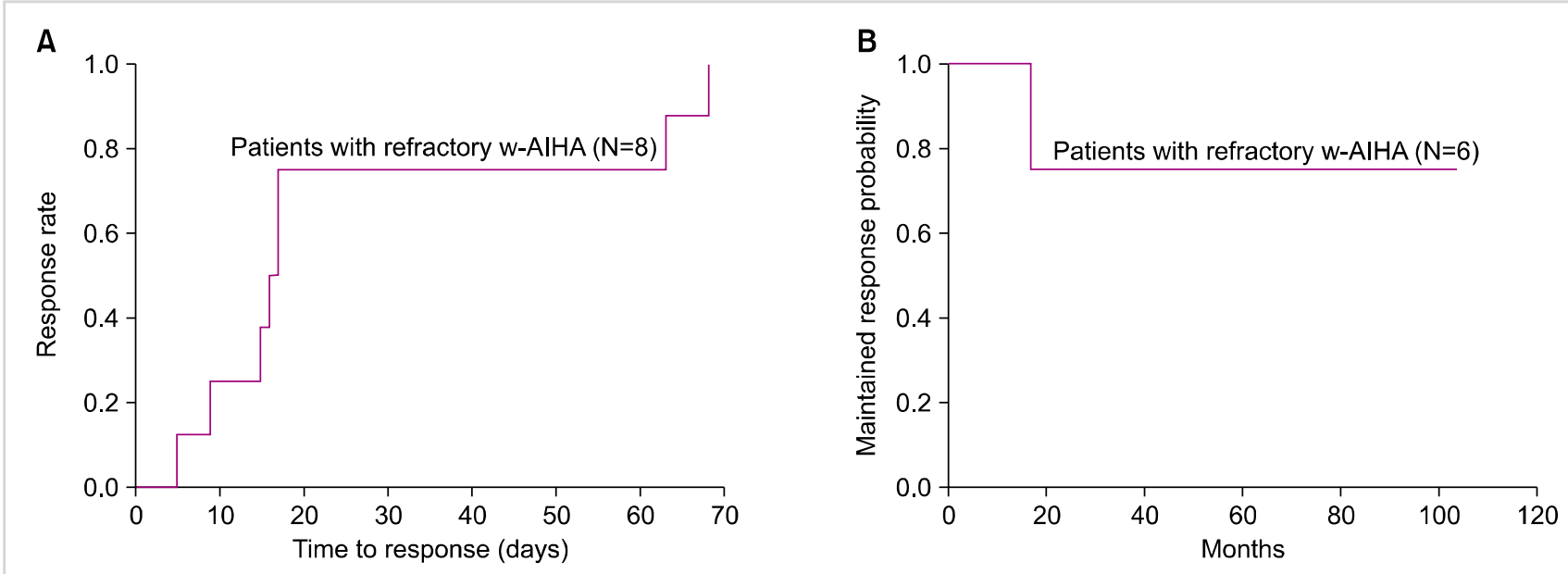

Fig. 3. Time to response (days) in 8 patients with refractory warm autoimmune hemolytic anemia treated with rituximab at $100 \mathrm{mg} / \mathrm{wk} / 4 \mathrm{weeks}$ (A). Maintained response (MR) probability in 6 patients with refractory w-AlHA treated with rituximab at $100 \mathrm{mg} / \mathrm{wk} / 4$ weeks (B). Two patients were excluded because MR was less than 6 months.

(100\%), intravenous gamma globulin (20\%), cyclophosphamide (50\%), danazol (20\%), and splenectomy (70\%); In five refractory patients, treatment with cyclophosphamide led to a transient response followed by relapse; patients responded to low doses of rituximab with MR at last follow-up at a median 100 (1-270) months.

Eight refractory patients were treated with rituximab at $100 \mathrm{mg} / \mathrm{wk}$ for 4 weeks plus HDD. The median time to administration was 14.8 (0.56-178.9) months from diagnosis of w-AIHA. An initial response was achieved in $100 \%$ of patients; three patients had a $\mathrm{CR}$ and five had a $\mathrm{PR}$, with a median time to response of 16 days (95\% CI, 14.1-17.8) (Fig. 3A). The probability of MR was $75 \%$ at 103 months; mean MR was $81.93 \pm 18$ months (Fig. $3 \mathrm{~B}$ ).

\section{DISCUSSION}

No evidence-based therapeutic regimen has been established for w-AIHA, and limited information on the response to treatment options is available, particularly for rituximab added to classic steroid frontline therapy at standard or low doses. In addition, the time to response in $\mathrm{w}$-AIHA varies considerably, from weeks to months $[16,17]$. In our patients, the time to response was 13.5 days. The main causes of this heterogeneity are not well established; one important factor could be related to the time to treatment initiation after first clinical manifestations of anemic syndrome.

Steroids are the standard first-line therapy in w-AIHA [18]. All patients in our cohort received this treatment, and 46 patients who received steroids alone had a response rate of $78 \%[6,7]$. RFS in these patients confirmed that steroids should remain the first-line treatment, as stated in the most recent published guideline [18].

Recently, it has been reported in some centers that rituximab at standard and low doses, plus steroids, could be effec- tive as first-line treatment in newly diagnosed AIHA [19, 20]. Furthermore, we previously showed that low-dose rituximab plus steroids as first choice therapy yields excellent results in newly diagnosed ITP $[8,9]$, which has a pathophysiology closely resembling that of w-AIHA. Based on this experience, we added rituximab at $100 \mathrm{mg} / \mathrm{wk}$ for 4 weeks to HDD as front-line therapy in 18 patients, achieving a response in $100 \%$; this is similar to a recently reported response of $93 \%$ [21], and $87.5 \%$ reported in a recent retrospective multicenter study at standard doses plus a fixed dose $1,000 \mathrm{mg}$ rituximab [22], as well as a rate of $75 \%$ with $375 \mathrm{mg} / \mathrm{m}^{2}$ plus prednisolone reported in another study [20]. Few studies have prospectively investigated the role of rituximab plus steroids as first-line treatment in AIHA. One study included eight patients with newly diagnosed AIHA treated with rituximab at low doses plus oral prednisone, achieving an ORR of $90 \%$ and RFS at 36 months of $67 \%$ vs. the $58.7 \%$ in our group. In another prospective study of 64 adult patients with AIHA treated with rituximab at $375 \mathrm{mg} / \mathrm{m}^{2}$ plus prednisolone, an RFS of $70 \%$ was achieved at 36 months vs. $58.7 \%$ in our patients; the findings of both these studies have been questioned by some authors [23] owing to the lack of long-term follow-up and the small number of patients. In the cited studies, rituximab was effective as first-line therapy in achieving a response and maintaining a higher RFS. Importantly, in our group, rituximab was administered at $20 \%$ of the standard dose with no adverse effects, in contrast to $37.5 \%$ of patients who experienced adverse effects in the previous report [20].

Rituximab at the standard dose of $375 \mathrm{mg} / \mathrm{m}^{2}$ is effective in cases of refractory AIHA, as second- to third-line therapy $[10,18]$. Recently, a guideline formally recommending rituximab as second-line therapy was issued [18], reflecting the current importance of rituximab as a nonsurgical option that is gradually replacing splenectomy in the treatment choice order for relapsed w-AIHA. 
In our cohort, 10 patients were refractory to a median of 2.5 different treatments; eight of them were treated with low doses of rituximab plus HDD and a response was achieved in all patients, with an MR probability of $75 \%$, similar to a recent report [12]. This finding confirmed that low doses of rituximab are effective in patients with refractory w-AIHA. We found no statistical difference in RFS between patients treated with steroids alone and those treated with low-dose rituximab plus steroids as first-line treatment. This leads us to conclude that prospective clinical trials involving a statistically powered group of patients and long-term follow-up are needed to evaluate front-line treatment in w-AIHA. Importantly, low-dose rituximab plus HDD was an effective salvage therapy in patients with refractory w-AIHA.

Splenectomy is the classic second-line therapy in w-AIHA [24]. In our group, seven $(10.8 \%)$ patients were treated this way, similar to $13 \%$ reported in other centers [6]. A response was achieved in all patients; nevertheless, they relapsed and were successfully treated as patients with refractory w-AIHA using low doses of rituximab plus HDD. Most of our patients rejected splenectomy, underscoring the decreasing use of this surgical procedure, as rituximab-based biological therapy becomes increasingly accepted. In this respect, clinical trials comparing splenectomy versus rituximab at standard and low doses in the long term are needed to define the precise indications for these treatment options and to justify a medical over surgical choice of therapy in w-AIHA.

The principal limitations in the present report are its retrospective design, limited number of patients, and the possibility that mostly patients with more active w-AIHA could have been referred to our center. In addition, serological characterization of the involved antibodies was not performed owing to a lack of monospecific Coombs antisera and reagent RBCs. The main strengths of this study are a clear documentation of the different treatment lines, adequate length of follow-up, and robust statistical analysis.

In conclusion, the response to standard doses of steroids and other lines of treatment in patients with w-AIHA varied considerably, whereas therapy with rituximab plus high doses of dexamethasone was effective in refractory patients. Further trials are needed to define the role of rituximab at standard or repeated low doses plus HDD as first-line treatment in w-AIHA.

\section{Authors' Disclosures of Potential Conflicts of Interest}

No potential conflicts of interest relevant to this article were reported.

\section{REFERENCES}

1. Jaime-Pérez JC, Rodríguez-Martínez M, Gómez-de-León A, Tarín-Arzaga L, Gómez-Almaguer D. Current approaches for the treatment of autoimmune hemolytic anemia. Arch Immunol Ther
Exp (Warsz) 2013;61:385-95.

2. Barros MM, Blajchman MA, Bordin JO. Warm autoimmune hemolytic anemia: recent progress in understanding the immunobiology and the treatment. Transfus Med Rev 2010;24: 195-210.

3. Anguiano-Álvarez VM, Hernández-Company A, Hamdan-Pérez $\mathrm{N}$, et al. Splenic myeloid metaplasia in warm autoimmune hemolytic anemia (wAIHA): a retrospective study. Blood Res 2018;53:35-40.

4. Barcellini W, Fattizzo B, Zaninoni A, et al. Clinical heterogeneity and predictors of outcome in primary autoimmune hemolytic anemia: a GIMEMA study of 308 patients. Blood 2014;124:2930-6.

5. Alonso HC, Manuel AV, Amir CG, et al. Warm autoimmune hemolytic anemia: experience from a single referral center in Mexico City. Blood Res 2017;52:44-9.

6. Roumier M, Loustau V, Guillaud C, et al. Characteristics and outcome of warm autoimmune hemolytic anemia in adults: New insights based on a single-center experience with 60 patients. Am J Hematol 2014;89:150-5.

7. Crowther M, Chan YL, Garbett IK, Lim W, Vickers MA, Crowther MA. Evidence-based focused review of the treatment of idiopathic warm immune hemolytic anemia in adults. Blood 2011;118:4036-40.

8. Gómez-Almaguer D, Colunga-Pedraza PR, Gómez-De León A, Gutiérrez-Aguirre CH, Cantú-Rodríguez OG, Jaime-Pérez JC. Eltrombopag, low-dose rituximab, and dexamethasone combination as frontline treatment of newly diagnosed immune thrombocytopaenia. Br J Haematol 2019;184:288-90.

9. Gómez-Almaguer D, Tarín-Arzaga L, Moreno-Jaime B, et al. High response rate to low-dose rituximab plus high-dose dexamethasone as frontline therapy in adult patients with primary immune thrombocytopenia. Eur J Haematol 2013;90:494-500.

10. Valent P, Lechner K. Diagnosis and treatment of autoimmune haemolytic anaemias in adults: a clinical review. Wien Klin Wochenschr 2008;120:136-51.

11. WHO. Haemoglobin concentrations for the diagnosis of anaemia and assessment of severity. Vitamin and Mineral Nutrition Information System. Geneva, Switzerland: World Health Organization, 2011. (Accessed Oct 2, 2018, at https://apps. who.int/iris/bitstream/handle/10665/85839/WHO_NMH_NH D_MNM_11.1_eng.pdf?ua=1).

12. Peñalver FJ, Alvarez-Larrán A, Díez-Martin JL, et al. Rituximab is an effective and safe therapeutic alternative in adults with refractory and severe autoimmune hemolytic anemia. Ann Hematol 2010;89:1073-80.

13. Zanella A, Barcellini W. Treatment of autoimmune hemolytic anemias. Haematologica 2014;99:1547-54.

14. Lechner K, Jäger U. How I treat autoimmune hemolytic anemias in adults. Blood 2010;116:1831-8.

15. Barcellini W. Current treatment strategies in autoimmune hemolytic disorders. Expert Rev Hematol 2015;8:681-91.

16. D'Arena G, Califano C, Annunziata M, et al. Rituximab for warm-type idiopathic autoimmune hemolytic anemia: a retrospective study of 11 adult patients. Eur J Haematol 2007; 79:53-8.

17. Gupta N, Kavuru S, Patel D, et al. Rituximab-based chemotherapy for steroid-refractory autoimmune hemolytic anemia of chronic 
lymphocytic leukemia. Leukemia 2002;16:2092-5.

18. Hill QA, Stamps R, Massey E, et al. The diagnosis and management of primary autoimmune haemolytic anaemia. $\mathrm{Br} \mathrm{J}$ Haematol 2017;176:395-411.

19. Barcellini W, Zaja F, Zaninoni A, et al. Sustained response to low-dose rituximab in idiopathic autoimmune hemolytic anemia. Eur J Haematol 2013;91:546-51.

20. Birgens H, Frederiksen H, Hasselbalch HC, et al. A phase III randomized trial comparing glucocorticoid monotherapy versus glucocorticoid and rituximab in patients with autoimmune haemolytic anaemia. Br J Haematol 2013;163:393-9.

21. Bussone G, Ribeiro E, Dechartres A, et al. Efficacy and safety of rituximab in adults' warm antibody autoimmune haemolytic anemia: retrospective analysis of 27 cases. Am J Hematol 2009; 84:153-7.

22. Serris A, Amoura Z, Canouï-Poitrine F, et al. Efficacy and safety of rituximab for systemic lupus erythematosus-associated immune cytopenias: A multicenter retrospective cohort study of 71 adults. Am J Hematol 2018;93:424-9.

23. Rodrigo C, Rajapakse S, Gooneratne L. Rituximab in the treatment of autoimmune haemolytic anaemia. Br J Clin Pharmacol 2015;79:709-19.

24. Sys J, Provan D, Schauwvlieghe A, Vanderschueren S, Dierickx D. The role of splenectomy in autoimmune hematological disorders: Outdated or still worth considering? Blood Rev 2017; 31:159-72. 